\title{
Isolated spina bifida
}

INSERM

\section{Source}

INSERM. (1999). Orphanet: an online rare disease and orphan drug data base. Isolated spina bifida. ORPHA:823

Spina bifida is the most common of a group of birth defects called neural tube defects.

The neural tube is the embryonic structure that develops into the brain and spinal cord.

Often called open spine, spina bifida affects the backbone and, sometimes, the spinal cord. Aperta spina bifida defines the dorsal malclosure of vertebrae, associated with various degrees of spine defects. A pocket of skin may form, containing meninges (meningocele) or spinal cord and meninges (myelomeningocele). 